\title{
SRSF2 Gene Deletion
}

National Cancer Institute

\section{Source}

National Cancer Institute. SRSF2 Gene Deletion. NCI Thesaurus. Code C133699.

A molecular abnormality referring to the loss of at least one copy of the SRSF2 gene. 